\title{
Web Technologies and Multi-criteria Analysis Used in Enterprise Integration
}

\author{
Marius CIOCA ${ }^{1}$, Lucian - Ionel CIOCA ${ }^{1}$, Luminita DUTA ${ }^{2}$ \\ 1 Lucian Blaga University of Sibiu, \\ 10, Bd-ul Victoriei, Sibiu, 550024, ROMANIA \\ 2 VALAHIA University of Targoviste, \\ 2, Bd-ul Carol I, 130024, Targoviste, ROMANIA
}

\begin{abstract}
The studies, research and solutions presented below started from the acute need of small and medium-sized enterprises to integrate their processes, services and relationships with customers and suppliers, in a cost-effective manner, given the fact that ERP systems are quite expensive for such organizations.
\end{abstract}

Keywords: Web technologies, multi-criterion analysis, modelling languages, enterprise integration.

\section{Introduction}

The survival and development of Romanian enterprises require alignment with international norms on product and service quality. First and foremost, all top manufacturers and importers require that the products they are supplied with be produced in systems that facilitate their improvement in terms of quality, environment, health, and occupational safety, etc.

This paper aims to create/present a methodology for the integration of the enterprise and its departments as well as the integration of the enterprise with its suppliers and customers in order to answer promptly to changes on the market. The model does not aim at developing a system able to be implemented - from scrap - into an enterprise, but to focus on the integration of the already extant systems (departments, human resources, software, hardware, etc.), because of the lower implementation costs than those involved in the case of a specialized ERP systems, such as SAP (a system which can be purchased by multinational enterprises only, because of its high costs).

\section{Current Status}

In Romania, the development of the infrastructure, of the communication and information systems and services, is a mandatory requirement for the overall economic development, as well as for the integration into globalized economy, and especially in the economic and social environment of the EU economy.
The approach is meant to support small and medium-sized enterprises in their development and in adapting to the new "ebusiness" and "e-commerce" environment, in achieving better communication standards within an organization, which is no longer confined within "four-walls", but extends on a national, European and worldwide level, in using the web and Internet technologies which help (with low costs) Romania meet the recent EU requirements related to an "eEurope” [1], [13].

\section{The Study of the Modeling Languages through Multi- attribute Decision Analysis}

In order to perform a multi-criterion analysis we have used the methodology approached in the comparative analysis of reference architectures presented in [3], [5], [8].

For this multi-attribute analysis we selected the following modeling languages: GRAI/GIM, Petri Nets, OOA/OMT, CIMOSA, IDEF0, IDEF1X, IDEF3, IEM, ARIS, EXPRESS and UML.

These representative techniques and methods are presented below.

GRAI and GRAI/GIM (Graph with Results and Activities Interrelated/GRAI Integrated Methodology). Its proposed methods are static descriptive models [7];

Petri Nets. These [11] are graphical and mathematical tools for representing and analyzing discrete event systems, characterized by parallelism and synchronization. Modeling enterprises by 
using Petri networks can be done from the following perspectives: functional, informational and resource using extensions of these networks. A very good example in this regard is the work of Neagu [9] which presents formalism and the use of the Petri networks in detail;

OOA (Object Oriented Modeling) and OMT. Object-oriented methods are increasingly used in enterprise modeling [5];

CIMOSA (Computer Integrated Manufacturing - Open System Architecture) covers, in addition to functional, informational and resource aspects the organizational aspects of the enterprise at different levels, thanks to the detailed model [14];

IDEF0 (Integrated Definition). The IDEF0 Language is based on the SADT (Structured Analysis and Design Technique) technique, developed by Douglas T. Ross and SofTech, Inc. and it is used for modeling functional aspects. Its aim was to develop generic subsystems to be used by enterprises in order to achieve a substantial improvement of the the industry as a whole [4];

IDEF1X. The Informational Modeling technique IDEF1X is used to model data in a standard, consistent, predictable manner, to manage data as a resource;

IDEF3 is a graphical modeling method used to facilitate the description of processes. It is composed of a limited set of constructions to describe the steps of the process (termed as units of behavior) and their connections. The resulted models are static descriptive models. However, they can be expanded and translated into Petri networks for analysis and simulation purposes;

IEM (Integrated Enterprise Modeling) covers the informational and functional perspectives of the enterprise. It is based on an objectoriented modeling approach, in which the central structure is a IDEF0 block in which all inputs and outputs are state objects of three types: commands, products and resources;

ARIS (Architecture for Information System) is a product of Prof. Scheer GmbH, modeling the enterprise from a functional, informational and organizational perspective;
EXPRESS is based on Chen's ER model (Entity Relationship) to specify STEP entities (ISO 10303-11:1994);

UML (Unified Modeling Languages). The unified modeling language is a language for building, specifying, visualizing and documenting software products. It is supported by numerous CASE tools, of which Rational Rose is the most representative. Because it is a simple and expressive language, of visual modeling, independent of programming languages, flexible, and because of the growing market for $\mathrm{OO}$ (object oriented) tools, this language is becoming increasingly used in modeling systems as well as enterprise modeling [2].

Thus, in order to select the best modeling language recommended for the EMLs (Enterprise Modeling Languages) block of the GERAM architecture, we have analyzed, based on the literature [4], [11], [12], [14] etc, the following modeling languages GRAI/GIM, Petri networks, OOA/OMT, CIMOSA, IDEF0, IDEF1X, IDEF3, IEM, ARIS, EXPRESS and UML which form the alternative set $A=\{A 1, A 2$, , A11 $\}$. [6], [7].

In order to establish the evaluation criteria presented below, we considered the modeling requirements that modeling languages have to meet. [9], [10]. Thus, for the selection of the modeling language based on the multi-attribute analysis 10 criteria have been defined, i.e. the set $E=\{E 1, E 2, \ldots, E 10\}$ as follows:

- From the point of view of the modeling and simulation support:

$$
\begin{aligned}
& \text { - } \quad \text { E1 - CASE tools; } \\
& \text { - E2 - methodological support; }
\end{aligned}
$$

- From the point of view of the modeling perspectives:

- E3 - the function perspective;

- $\quad \mathrm{E} 4$ - the information perspective;

- $\quad$ E5 - the resource perspective;

- $\quad$ E6 - the organization perspective;

- From the point of view of the modeling and coverage principles:

- E7 - distinguishing between problems;

- E8 - functional decomposition;

- E9 - genericity;

- E10 - distinguish between functionalities; 
The weight for every criterion is in this order $\{0.20,0.20,0.10,0.10,0.10,0.10,0.5,0.5$, $0.5,0.5\}$, where the set of weights form vector $\mathrm{w}$, which meets the requirement: the sum of all weights is 1 .

The scores employed in the multi-attribute analysis are cardinal, and range between 1 and 5.

The table of consequences resulted after the use of the established score for each criterion, in the case of each alternative is shown in the Table 1. $r_{i j}=\frac{\left(s_{i j}-s_{m j}\right)}{\Delta s_{j}}$

where: $\Delta s_{j}$ is the variation range defined by extreme scores $\left(\mathrm{s}_{\mathrm{mj}}\right.$ and $\left.\mathrm{s}_{\mathrm{Mj}}\right)$ of the alternatives considered, and is calculated according to the formula:

$$
\Delta s_{j}=s_{M j}-s_{m j} \text {, }
$$

Pairs $\left(\mathrm{s}_{\mathrm{mj}}\right.$ and $\left.\mathrm{s}_{\mathrm{Mj}}\right)$ of the lower values and the highest considering criterion $\mathrm{E}_{\mathrm{j}}$ are:

Table 1. The table of consequences for the problem of selecting a modeling language

\begin{tabular}{|c|c|c|c|c|c|c|c|c|c|c|}
\hline $\begin{array}{l}\text { Alternative } \\
\text { Criteria }\end{array}$ & E1 & E2 & E3 & E4 & E5 & E6 & E7 & E8 & E9 & E10 \\
\hline A1 & 3 & 5 & 5 & 5 & 5 & 5 & 5 & 5 & 5 & 5 \\
\hline A2 & 5 & 5 & 2 & 4 & 4 & 5 & 2 & 2 & 5 & 5 \\
\hline A3 & 5 & 5 & 5 & 5 & 5 & 2 & 2 & 2 & 2 & 2 \\
\hline A4 & 5 & 5 & 2 & 2 & 2 & 5 & 5 & 2 & 5 & 2 \\
\hline A5 & 3 & 5 & 5 & 4 & 5 & 5 & 3 & 2 & 5 & 5 \\
\hline A6 & 3 & 5 & 2 & 5 & 5 & 5 & 5 & 5 & 5 & 1 \\
\hline A7 & 5 & 4 & 2 & 4 & 5 & 5 & 5 & 2 & 5 & 2 \\
\hline A8 & 5 & 5 & 2 & 2 & 5 & 5 & 3 & 5 & 4 & 5 \\
\hline A9 & 5 & 5 & 2 & 2 & 5 & 2 & 4 & 5 & 4 & 5 \\
\hline A10 & 5 & 2 & 5 & 4 & 5 & 5 & 5 & 5 & 5 & 2 \\
\hline A11 & 5 & 5 & 2 & 2 & 5 & 5 & 4 & 5 & 4 & 5 \\
\hline
\end{tabular}

Table 2. The decision table for the problem of selecting a modeling language

\begin{tabular}{|c|c|c|l|l|l|l|l|l|l|l|c|c|}
\hline $\begin{array}{l}\text { Alternative } \\
\text { Criteria }\end{array}$ & E1 & E2 & E3 & E4 & E5 & E6 & E7 & E8 & E9 & E10 & $\begin{array}{l}\text { Final } \\
\text { score }\end{array}$ & $\begin{array}{c}\text { Final } \\
\text { classification }\end{array}$ \\
\hline & $\Delta_{1}=2$ & $\Delta_{2}=3$ & $\Delta_{3}=3$ & $\Delta_{4}=3$ & $\Delta_{5}=3$ & $\Delta_{6}=3$ & $\Delta_{7}=3$ & $\Delta_{8}=3$ & $\Delta_{9}=3$ & $\Delta_{10}=4$ & & \\
\hline $\mathrm{A} 1$ & 0.00 & 1.00 & 1.00 & 1.00 & 1.00 & 1.00 & 1.00 & 1.00 & 1.00 & 1.00 & 0.80 & $\mathrm{I}$ \\
\hline $\mathrm{A} 2$ & 1.00 & 1.00 & 0.00 & 0.67 & 0.67 & 1.00 & 0.00 & 0.00 & 1.00 & 1.00 & 0.73 & $\mathrm{IV}$ \\
\hline $\mathrm{A} 3$ & 1.00 & 1.00 & 1.00 & 1.00 & 1.00 & 0.00 & 0.00 & 0.00 & 0.00 & 0.25 & 0.71 & $\mathrm{~V}$ \\
\hline $\mathrm{A} 4$ & 1.00 & 1.00 & 0.00 & 0.00 & 0.00 & 1.00 & 1.00 & 0.00 & 1.00 & 0.25 & 0.61 & $\mathrm{IX}$ \\
\hline $\mathrm{A} 5$ & 0.00 & 1.00 & 1.00 & 0.67 & 1.00 & 1.00 & 0.33 & 0.00 & 1.00 & 1.00 & 0.68 & $\mathrm{VI}$ \\
\hline $\mathrm{A} 6$ & 0.00 & 1.00 & 0.00 & 1.00 & 1.00 & 1.00 & 1.00 & 1.00 & 1.00 & 0.00 & 0.65 & $\mathrm{VIII}$ \\
\hline $\mathrm{A} 7$ & 1.00 & 0.67 & 0.00 & 0.67 & 1.00 & 1.00 & 1.00 & 0.00 & 1.00 & 0.25 & 0.71 & $\mathrm{~V}$ \\
\hline $\mathrm{A} 8$ & 1.00 & 1.00 & 0.00 & 0.00 & 1.00 & 1.00 & 0.33 & 1.00 & 0.67 & 1.00 & 0.75 & $\mathrm{III}$ \\
\hline $\mathrm{A} 9$ & 1.00 & 1.00 & 0.00 & 0.00 & 1.00 & 0.00 & 0.67 & 1.00 & 0.67 & 1.00 & 0.67 & $\mathrm{VII}$ \\
\hline $\mathrm{A} 10$ & 1.00 & 0.00 & 1.00 & 0.67 & 1.00 & 1.00 & 1.00 & 1.00 & 1.00 & 0.25 & 0.73 & $\mathrm{IV}$ \\
\hline $\mathrm{A} 11$ & 1.00 & 1.00 & 0.00 & 0.00 & 1.00 & 1.00 & 0.67 & 1.00 & 0.67 & 1.00 & 0.77 & $\mathrm{II}$ \\
\hline Ponderi & 0.20 & 0.20 & 0.10 & 0.10 & 0.10 & 0.10 & 0.5 & 0.5 & 0.5 & 0.5 & & \\
\hline
\end{tabular}

The decision table is obtained by normalizing the scores presented above which is to be made through formulas 2 and 3 , and the final score obtained by each alternative is calculated according to formula 1 .

$$
J_{i}=\sum_{j=1}^{n} w_{j} r_{j},
$$

where: $\mathrm{w}$ is the weight vector $\mathrm{w}_{j}$ of criteria $\mathrm{E}_{\mathrm{j}}$ $(\mathrm{j}=1,1, \mathrm{nc})$, nc is the number of evaluation criteria and $r_{j}$ represents the normalized scores according to the formulas below:

$r_{i j}=\frac{\left(s_{M j}-s_{i j}\right)}{\Delta s_{j}}$, $\mathrm{s}_{\mathrm{mj}}=\min \left(\mathrm{s}_{\mathrm{ij} j}, \mathrm{i}=1,2, \ldots, \mathrm{na}\right) ; \mathrm{j}=1,2, \ldots, \mathrm{nc}$

$s_{M j}=\max \left(s_{i j}, i=1,2, \ldots, n a\right) ; j=1,2, \ldots, n c$

where: na is the number of alternatives, and nc is the number of evaluation criteria.

The graphic representation of the results in the table above is shown in Figure 1.

As shown above, the UML was ranked second. However, we have selected and recommended this language because of the following reasons:

- the UML integrates more easily with Web technologies used for the development of the proposed model. Thus, the data may be modeled by means 
of the UML and handled by means of the XML. In addition, it enables the user the generate Java code (program code);

- IT specialists dealing with industrial systems analysis and development are more "inclined" to work with the UML, unlike system analysts coming from other fields and who probably prefer IDEF or ARIS. enterprise at an intermediate level, specific to a particular sector and, ultimately, to the lowest (specific) level at which the model refers to a particular enterprise.

PERA Purdue Enterprise Reference Architecture (PERA) was designed to assist the industry in its development and implementation efforts of integrated production systems. It is a

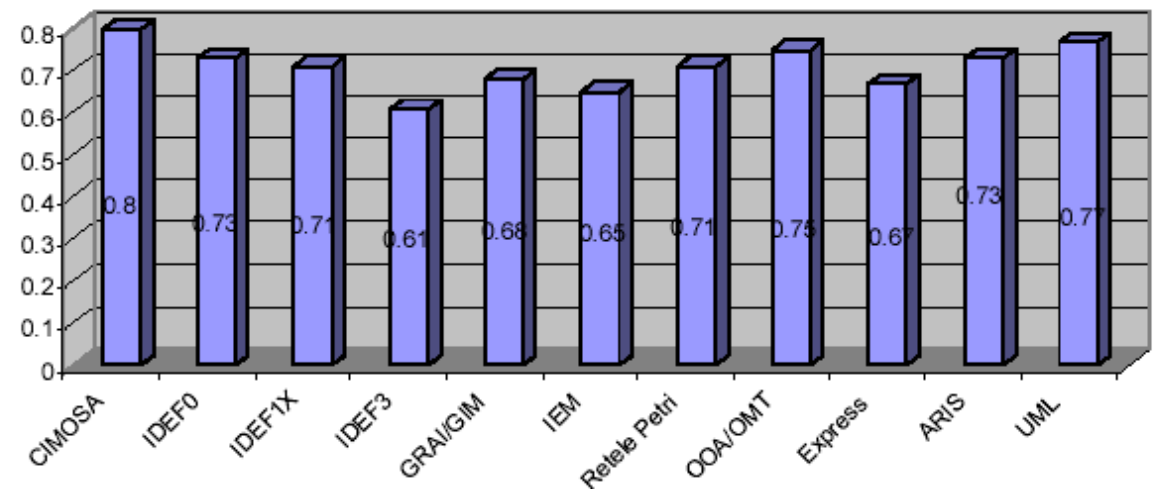

Figure 1. The classification of the modeling languages resulted from the decision table

\section{The Study of Information SystemsReference Architectures in the Industrial Environment}

The study of the enterprise integration reference architectures through multiattribute decision analysis may be employed by means of the method presented above and described in [3]. From all architectures in the literature (GERAM, CIMOSA, GRAI/GIM, PERA etc) it is recommended that GERAM be used for enterprise modeling.

CIMOSA In 1985, the ESPRIT program through the AMICE consortium lays the foundation for a CIM architecture for enterprise integration - CIMOSA (Computer Integrated Manufacturing - Open System Architecture). CIMOSA has three components:

- the enterprise modeling framework;

- the modeling language;

- the enterprise infrastructure.

CIMOSA does not only provide a reference model, but follows the modular model constructed in the integrated enterprise engineering environment. In a step by step approach, the degree of abstraction of the model is reduced from the general (generic) level, which is specific to any complete and widely documented methodology to define, design, build, install and operate an integrated enterprise system or a major automation project and was developed by Purdue University, USA, in collaboration with a consortium of industrial companies. This architecture is known in particular because of its main focus on the human aspect and its interactions with various aspects of automation.

GRAI/GIM (Graph with Results and Activities Interrelated/GRAI Integrated Methodology) was developed by the laboratory of the Bordeaux University (France). The reference model GRAI considers the production system as a set of three subsystems:

- the decision-making system;

- the information system;

- the physical system.

GRAI / GIM covers the entire life cycle of the production system except operation and decommissioning phases. The four perspectives differ from those of CIMOSA architecture, a decision-making and a physical perspective are introduced. The four perspectives used during the user-oriented analysis and design are converted into three perspectives of implementation during the technical-oriented design stage. 
IEM (Integrated Enterprise Modeling) [4] is a reference architecture that covers the functional and informational perspectives of the enterprise. It is based on an objectoriented modeling method in which the central structure is an IDEF0 (Integration DEFinition) block in which all inputs and outputs are object states of three categories:

- - commands;

- $\quad$ - products;

- - services.

ARIS performs a detailed description of an enterprise in terms of data model, functional, organizational and process models, as well as from the perspective of model analysis, it is an indispensable requirement for defining terms discussed in an enterprise. Some of these terms are:

- business process reengineering;

- effective selection and introduction of standard application systems;

- development of specialized software;

- development of architectural plans for information technology;

- documentation on the existing data processing method;

- workflow management.

ARIS architecture (Architektur integrierter Informationssystem) is strongly supported by software tools such as ARIS ToolSet, which includes components of modeling, analysis and navigation of user interfaces, functions and integrated database. Some of these features are:

- multi-user operating capacity;

- graphical user interface, object-oriented, under a Windows environment;

- relational database with automatic restoration;

- SQL/ODBC interface;

- management of reference models;

- assessments and comparisons between models;

- adapting the model, based on rules (starting from the reference model);

- easy display of the entire available information for each group of objects and for individual objects;
- wide range of browsing functions for complex enterprise models.

GERAM. Starting from the assessment of the most representative reference architectures for enterprise integration (CIMOSA, GRAI/GIM and PERA), the IFAC / IFIP workgroup have developed a global definition of a generalized architecture. The proposed framework was named GERAM (Generalized Enterprise Reference Architecture and Methodology). GERAM refers to those methods, models and tools that are necessary to build and operate the enterprise.

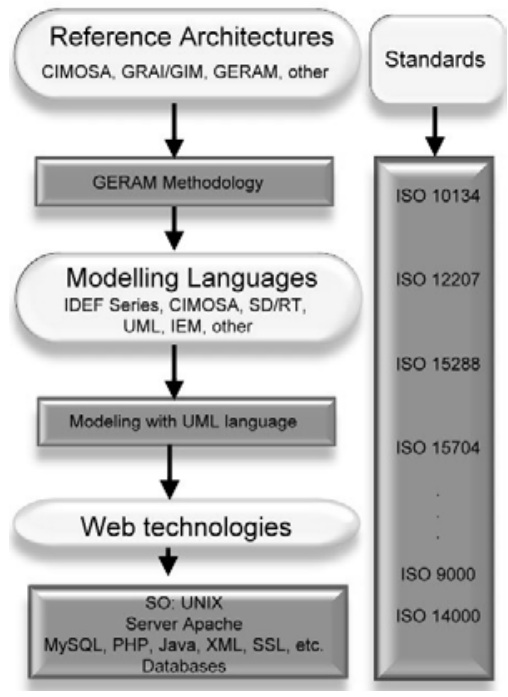

Figure 2. Elements of enterprise integration

GERAM provides a description of all the elements recommended in engineering and enterprise integration, thus establishing a standard for the collection of tools and methods enterprise will benefit from in order to successfully manage initial integration design and of the change processes that may occur during life cycle of enterprise operation. GERAM does not impose a particular set of tools and methods, but defines the criteria that must be met by such a combination.

\section{Conclusions}

In conclusion, we have come to the following:

Where, in order to cut costs, a small or medium-sized enterprise should use [2]:

- the GERAM architecture for enterprise modeling;

- the UML is the recommended language for enterprise modeling and for "filling" the 
EMLs (Enterprise Modeling Languages) block in the GERAM architecture;

- the recommended ISO standards should be taken into account during the development of the enterprise integration model/methodology;

- For the integration of the enterprise both within its "four walls", but especially with its suppliers and customers as well as with its subsidiaries, opensource Web technologies should be used, due to the fact that they involve not costs (licenses are free) and they are flexible and established. From this point of view, we recommend the following web technologies: the PHP application server, the MqSQL/PostgreSQL database server, Java, JavaScript, the Apache Web server running on Linux as well as the XML language for data handling within and outside the organization.

\section{REFERENCES}

1. BURAGA, S. C, M. CIOCA, Using Semantic Web Technologies to Enhance the Inter-Connectivity Between the Components of an ELearning System, 4th Intl. Conf. on RoEduNet Romania, May 20-22, 2005 Targu Mures ROMANIA, pp. 17-18, ISBN: 978-973-7794-29-1;

2. CIOCA, M., S. C. BURAGA, New Tools for Human Resource Management in e-Business: Combining UML Language, Reference Architectures and Web Programming, IEEE Intl. Conf. on Industrial Informatics, Aug. 2124, 2003 Alberta CANADA, pp. 170174, ISBN: 0-7803-8200-5;

3. CIOCA, M., LI. CIOCA, Multi-criterion Analysis of Reference Architectures and Modeling Languages used in Production Systems Modeling, 3rd IEEE Intl. Conf. on Industrial Informatics (INDIN), Aug. 10-12, 2005, Perth AUSTRALIA, pp. 230-233, ISBN: 07803-9094-6;

4. FILIP, F. G., B. BARBAT, Informatica industriala: Noi paradigme si aplicatii, Editura Tehnica, Bucuresti, 1999;
5. FILIP, F. G, G. NEAGU, Object Oriented Approach to Software Engineering for CIME, in P. Groumpos (Ed.). Preprint, 8th IFAC/IFIP/IFORS/IMACS/IFIP Symp. "Large Scale Systems: Theory and Applications”, Patras (July 15-17), 9, 1994, pp. 400-404;

6. ISO 10303-11:1994 Industrial Automation Systems and Integration Product data representation and exchange. Part 1: Overview and fundamental principles;

7. KOSANKE, K., Comparison of Enterprise Modeling Methodologies. in J. Goosenaerts, F. Kimura, H. Wortmann (Eds.) Information Infrastructure Systems for Manufacturing. Champman \& Hall, London, 1997, pp. 115-127;

8. KROGSTIE, J., S. ARNESEN, Comparing Languages for Enterprise Modeling using a Language Quality Framework, Norwegian University of Science and Technology, Institute of Computer and Information Sciences, 2003;

9. NEAGU, G. Contributii privind conceptia si metodele de rezolvare a sistemelor pentru conducerea atelierelor de fabricatie, Teza de doctorat, Universitatea Politehnica din Bucuresti, 1998;

10. OPREAN, C., C. V. KIFOR, Modelling the Implementation, Usage and Maintenance of the ISO 9000 Quality Management System using the Reference Architectures. in TMCM 1998, Iasi, Romania;

11. REISIG, W. Petri Nets. Springer Verlag, Berlin, 1985;

12. RUTAKYAMIRWA, N. N., Modeling of Business Process in Distributed Environment: a Case Study of Issuance of Certificate of Occupancy in Tanzania, 2002;

13. SUDUC, A., M. BIZOI, F. FILIP, User Awareness about Information Systems Usability, Studies in Informatics and Control, Vol. 19(2), June 2010;

14. WILLIAMS, TJ et al, Architectures for Integration Activities and Enterprises, Computer in Industry, 24, 1994, pp. 111-139. 